1989

\title{
On the evolution of intensive cyclonic-anticyclonic vortex pairs
}

\author{
Konshin, VN
}

http://hdl.handle.net/10026.1/9757

10.1016/s0422-9894(08)70219-8

Elsevier

All content in PEARL is protected by copyright law. Author manuscripts are made available in accordance with publisher policies. Please cite only the published version using the details provided on the item record or document. In the absence of an open licence (e.g. Creative Commons), permissions for further reuse of content should be sought from the publisher or author. 


\section{MESOSCALE/SYNOPTIC COHERENT STRUCTURES IN GEOPHYSICAL}

\section{TURBULENCE}

PROCEEDINGS OF THE 20TH INTERNATIONAL LIEGE COLLOQUIUM ON OCEAN HYDRODYNAMICS

\section{Edited by}

J.C.J. NIHOUL

University of Liège, B5 Sart Tilman, B-4000 Liège, Belgium

and

\section{B.M. JAMART}

MUMM, Institute of Mathematics, 15 Avenue des Tilleuls, B-4000 Liège, Belgium

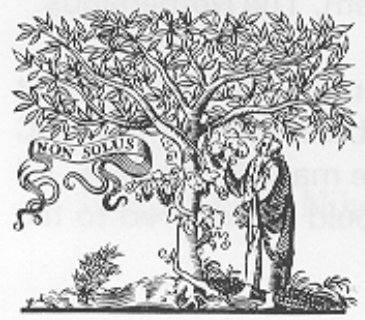

\section{ELSEVIER}

Amsterdam - Oxford - New York - Tokyo 1989 


\title{
ON THE EVOLUTION OF INTENSIVE CYCLONIC-ANTICYCLONIC VORTEX PAIRS
}

\author{
G.I. SHAPIRO \\ P.P. Shirshov Institute of Oceanology, USSR Academy of Sciences, 23 Krasikova Street, \\ 117218 Moscow, USSR
}

V.N. KONSHIN

Institute of Computer Aided Design, USSR Academy of Sciences, 40 Vavilova Street, 117333 Moscow, USSR

\begin{abstract}
Intensive baroclinic eddies in the ocean are shown to have a new type of nonlinearity which is not taken into account by the traditional quasi-geostrophic equation. The evolution and decay of dipole eddies are investigated using a generalized near-geostrophic model. The time interval after which the traditional approach is no longer valid is estimated analytically. Two sets of numerical calculations are presented. In the first one, the evolution of "modons" is simulated and shown to depend on their intensity and size. It is found that additional nonlinear effects change the trajectory of relatively small eddies rather than their structure. In the second set of experiments, we consider the process of coupling of initially monopole eddies in a shear flow.
\end{abstract}

\section{INTRODUCTION}

Mesoscale dipole eddies have been observed in many parts of the World Ocean. They are often referred to as "mushroom-like currents", a term introduced by Professor Konstantin Fedorov (Ginsburg and Fedorov, 1984). Highly organized cyclonic-anticyclonic pairs can also be realized in laboratory experiments (Ginsburg et al., 1987; Flierl et al., 1983). It has been shown theoretically in the work of Flierl et al. (1983) that any slowly varying and isolated disturbance in a stratified fluid on a $\beta$-plane must have zero net relative angular momentum, so that the dipole is one of the simplest dynamically consistent representation of such a disturbance. Analytical models of dipole eddies based on the solution of the Quasi-Geostrophic Equation (QGE) have been presented by Stern (1975), Larichev and Reznik (1976), and others.

In this paper some aspects of the evolution of cyclonic-anticyclonic vortex pairs are investigated mainly by numerical simulation. The present level of theoretical knowledge and the available observational data do not allow to give a proper numerical forecast of the behaviour of real ocean mesoscale eddies for a durable period of time of the order of several years. Thus, theoreticians concentrate their attention on the detailed study of certain idealized situations which are useful to understand the physical picture of the dynamical processes and to obtain reasonable estimates of the lifetime and trajectories of eddies. 
The observational data (Kamenkovich et al., 1982; Belkin et al., 1986; Ivanov et al., 1986) show that, for intense baroclinic eddies, the thickness of the fluid layer confined between two fixed isopycnal surfaces deviates significantly from the equilibrium state. This results in finite amplitude disturbances of the density field and Brunt-Vaisala frequency. The question then arises as to whether there are any qualitatively new features in the behaviour of eddies of finite amplitude in comparison with less intensive eddies. Eddies associated with small fluctuations of the density field are often described analytically by the QGE (Pedlosky, 1979; Kamenkovich et al., 1986) which is suitable to investigate the evolution of barotropic and baroclinic synoptic scale eddies. However, as follows from its derivation, this equation is not adequate for the description of disturbances with large amplitude fluctuations of the density field. In this case, one can use the so-called Generalized Near-geostrophic Equation (GNE) derived by Williams (1985) and Shapiro $(1986,1989)$. The GNE has no restrictions on the amplitude of the isopycnals displacement. This equation filters out high-frequency gravity waves, as does the traditional QGE, and it also includes some additional nonlinear terms.

In this paper, we consider intense baroclinic eddies on a $\beta$-plane using the reduced gravity approximation. It is assumed that the motion is sufficiently slow that $\mathrm{f} \mathrm{T}_{*} \gg 1$ (where $f=f_{0}+\beta y$ is the Coriolis parameter and $T_{*}$ is the time scale for the eddy evolution) and that the geostrophic balance dominates, so that $\mathrm{Ki}=\frac{\mathrm{u}_{*}}{\mathrm{f}_{0} \mathrm{~L}_{*}} \ll 1$, where $\mathrm{Ki}$ is the Kibel-Rossby number, $\mathrm{u}_{*}$ the current velocity scale and $\mathrm{L}_{*}$ the length scale (e.g., the radius of an eddy). In nondimensional variables, the GNE on a $\beta$-plane is reduced to:

$$
\mathrm{M} \frac{\partial \mathrm{H}}{\partial \mathrm{t}}=\mathrm{F}
$$

where

$$
\mathrm{F}=-J\left[(1+\varepsilon H) \nabla^{2} \mathrm{H}+\frac{1}{2} \varepsilon|\nabla H|^{2}, \mathrm{H}\right]+\frac{1+\varepsilon H}{(1+\varepsilon y)^{2}} \frac{\partial \mathrm{H}}{\partial \mathrm{x}}
$$

$\mathrm{M}=\mathrm{E}-\nabla[(1+\varepsilon \mathrm{H}) \nabla]$ is a nonlinear operator, $\mathrm{E}$ is the unit operator, $\nabla$ is the horizontal gradient operator, $H(x, y, t)=\frac{h-h_{*}}{\varepsilon h_{*}}$ is the nondimensional and $h$ the dimensional thickness of the dynamically active layer, $\mathrm{h}_{*}=$ const. being its typical value, $J$ is the Jacobian operator, $\varepsilon=\frac{\beta L_{R}}{f_{0}}$ is the sphericity parameter, and $L_{R}=\frac{\left(g^{\prime} h_{*}\right)^{1 / 2}}{f_{0}}$ is the internal radius of deformation. The horizontal distances and the time are nondimensionalized by the scales $L_{R}$ and $\left(\beta L_{R}\right)^{-1}$, respectively. The parameter $\varepsilon$ is assumed to be small on a $\beta$-plane. The nondimensional variables $\mathrm{H}, \mathrm{x}, \mathrm{y}, \mathrm{t}$ are not necessarily of order of one. It can be found from the geostrophic balance that the scale of the amplitude of the $H$ fluctuation is $H_{*}=\frac{u_{*} f_{0} L_{*}}{\varepsilon g^{\prime} h_{*}}$. Then the KibelRossby number can be rewritten as $\mathrm{Ki}=\frac{\varepsilon \mathrm{H}_{*}}{l^{2}}$, where $l=\frac{\mathrm{L}_{*}}{\mathrm{~L}_{\mathrm{R}}}$ is the nondimensional size. 
For $\varepsilon=0$, the GNE reduces to the QGE

$$
\frac{\partial}{\partial \mathrm{t}}\left(\Psi-\nabla^{2} \Psi\right)+J\left(\nabla^{2} \Psi, \Psi\right)-\frac{\partial \Psi}{\partial \mathrm{x}}=0
$$

where the function $\Psi$ is introduced instead of $H$. For $\varepsilon \neq 0$, the terms including $H$ in (1.1) describe the nonlinear effects caused by the finite amplitude displacement of the isopycnals.

To capture some new effects of the behaviour of dipole eddies, it is useful to consider initial conditions corresponding to stationary solutions (Rossby solitons) of the QGE. Then, any departure from the prescribed stationary motion of the dipole eddy should be caused by some additional effects which are not taken into account in the QGE. Even when the nonlinearity is small but acting over a long time, such effects result in a notable departure from the soliton solution and their role should not be neglected.

This paper is organized as follows. In Section 2, an analytical estimation is made of the time interval during which the soliton theory based on the QGE is applicable. In Section 3, we present an efficient finite-difference scheme for solving the GNE. The results of numerical simulations of the evolution and decay of vortex pairs are presented in Section 4. In Section 5, we investigate the processes of coupling and interaction of a cyclone and an anticyclone in a shear flow. The conclusions are summarized in Section 6.

\section{ANALYTICAL ESTIMATION}

The quasi-geostrophic equation (1.2) has exact solitary solutions of different types (Larichev and Reznik, 1976; Berestov and Monin, 1980; Berestov, 1981). A helpful review is presented in the monograph by Kamenkovich et al. (1986). The simplest example is the socalled Larichev-Reznik soliton or modon (Larichev and Reznik, 1976, McWilliams et al., 1981, Makino et al., 1980). A modon consists of two connected eddies of cyclonic and anticyclonic spin, the center of mass moving along the $\mathrm{x}$ axis with constant speed $\mathrm{c}$.

The Larichev-Reznik soliton is an exact solution of the QGE on a $\beta$-plane but is does not satisfy exactly more general equations such as the shallow water equations of the GNE. If such a soliton is taken as an initial condition, it would be changed in time under the action of finite amplitude effects which are not taken into account by the quasi-geostrophic model. The time interval during which the initial modon is approximately unchanged can be estimated using the GNE.

In a coordinate system $x^{\prime}=x-c t, y^{\prime}=y, t^{\prime}=t$ moving at constant zonal speed $c$, the GNE (1.1) can be written as:

$$
\begin{array}{r}
\frac{\partial \mathrm{H}}{\partial \mathrm{t}}=\mathrm{M}^{-1}\left\{\left[-J\left(\nabla^{2} \mathrm{H}, \mathrm{H}\right)+\frac{\partial \mathrm{H}}{\partial \mathrm{x}}(1+\mathrm{c})-\mathrm{c} \nabla^{2} \frac{\partial \mathrm{H}}{\partial \mathrm{x}}\right]-\mathrm{c} \varepsilon \nabla\left[\mathrm{H} \nabla \frac{\partial \mathrm{H}}{\partial \mathrm{x}}\right]\right. \\
\left.-\varepsilon J\left[\mathrm{H} \nabla^{2} \mathrm{H}+\frac{1}{2}|\nabla \mathrm{H}|^{2}, \mathrm{H}\right]+\frac{\partial \mathrm{H}}{\partial \mathrm{x}}\left[\frac{1+\varepsilon \mathrm{H}}{(1+\varepsilon y)^{2}}-1\right]\right\}
\end{array}
$$


where $\mathrm{M}^{-1}$ is the inverse operator to $\mathrm{M}$ and the primes are omitted.

The time derivative of $\mathrm{H}$ at any fixed time can be determined from (2.1) if the spatial distribution of $\mathrm{H}$ is known. Taking the Larichev-Reznik solution $\mathrm{H}=\mathrm{H}_{\mathrm{c}}$ as an initial condition and substituting it into the right-hand side of (2.1), we can calculate $\frac{\partial \mathrm{H}}{\partial \mathrm{t}}$ at $\mathrm{t}=0$. Note that some of the terms vanish. Further evaluation of the derivatives by ratios of appropriate scales gives the ordering relation:

$$
\frac{1}{\tau} \max \left[1, \frac{1+\varepsilon \mathrm{H}_{*}}{l^{2}}\right]=\varepsilon \max \left[\frac{\mathrm{cH}_{*}}{l^{3}}, \frac{\mathrm{H}_{*}^{2}}{l^{4}}, \frac{\mathrm{H}_{*}}{l}, 1\right]
$$

where $\mathrm{H}_{*}$ is the nondimensional scale for $\mathrm{H}_{c}$ (e.g., its maximum value), and $\tau$ is the nondimensional time scale for the evolution of the soliton, i.e., the time period during which the exact solution of the GNE does not deviate significantly from the Larichev-Reznik solution. The estimation of the soliton propagation speed $\mathrm{c}$ follows from (1.2) by the ordering relations:

$$
\mathrm{c}=\frac{\max \left(1, \frac{\mathrm{H}_{*}}{l^{3}}\right)}{\max \left(1, \frac{1}{l^{2}}\right)}
$$

This estimation is in good agreement with the results of the calculation of c versus $\mathrm{H}_{*}$ and $l$ made by McWilliams et al. (1981). The values of $c$ and $\tau$ depend on the specific relations between $\varepsilon, l$, and $\mathrm{H}_{* .}$ It follows from (2.2) and (2.3) that in the case $1<l<\frac{1}{\varepsilon}, l^{3}<\mathrm{H}_{*}<\frac{1}{\varepsilon}$ (the Kibel-Rossby number is in the range $\varepsilon l<\mathrm{Ki}<\frac{1}{l^{2}}$ ), the values of $\mathrm{c}$ and $\tau$ are:

$$
\mathrm{c}=\frac{\mathrm{H}_{*}}{l^{3}}, \quad \tau=\frac{l^{4}}{\varepsilon \mathrm{H}_{*}^{2}}=\frac{\varepsilon}{\mathrm{Ki}^{2}}
$$

We choose the following values as being typical for mid-latitude eddies: $\varepsilon=0.01, l=2$ and $\varepsilon \mathrm{H}_{*}=0.5$, so that the maximal disturbance in the thickness of a fluid layer is equal to $50 \%$. From (2.4), we find $c \approx 6, \tau \approx 0.6$, and $X=c \tau=4$ where $X$ is the zonal displacement of the eddy after time $\tau$. In dimensional variables, $X=4 \mathrm{~L}_{R}$, so that it is twice as large as the radius of the eddy. For other relationships between the parameters, the problem is solved similarly.

What happens after the time interval $\tau$ : the decay of the vortex pair or the rearrangement of its inner structure, a change of its propagation speed or a deflection from a strongly zonal motion? To answer such a question, we have carried out a set of numerical experiments with dipole eddies. 


\section{FINITE-DIFFERENCE SCHEME}

The fast and accurate numerical solution of equation (1.1) presents difficulties in the inversion of the operator $\mathrm{M}$. We use a modification of the finite-difference scheme developed in the work of Konshin and Shapiro (1988) which is based on the idea of physical splitting.

A uniform finite-difference grid is introduced within the two-dimensional region of interest; the grid nodes are defined by

$$
\left\{x_{i}=i \Delta x, \quad i=1,2, \ldots N ; \quad y_{j}=j \Delta y, \quad j=1,2, \ldots P\right\}
$$

where $\Delta \mathrm{x}$ and $\Delta \mathrm{y}$ are the space steps; $\mathrm{N}$ and $\mathrm{P}$ are the numbers of grid points along the $\mathrm{X}$ and $\mathrm{Y}$ directions, respectively.

The numerical procedure consists of three steps.

1) The grid approximation $\mathrm{F}^{\mathrm{n}}$ of the right-hand side of (1.1) is calculated at the $\mathrm{n}$-th level. The Jacobian operator is calculated using Arakawa's scheme and the last term is approximated by central differences.

2) The value of $\frac{\partial H}{\partial t}$ is obtained from the equation:

$$
\mathbf{M}^{\mathrm{n}} \frac{\partial \mathrm{H}}{\partial \mathrm{t}}=\mathrm{F}^{\mathrm{n}}
$$

where $\mathrm{M}^{\mathrm{n}}$ is the grid approximation of $\mathrm{M}$ at the $\mathrm{n}$-th level. It can be proved that $\mathrm{M}$ is an elliptical and self-adjoint operator (Konshin, 1984). To solve (3.2), we use a fast and economic iterative procedure:

$$
\frac{1}{\mu} \mathrm{R}\left[\left(\frac{\partial \mathrm{H}}{\partial \mathrm{t}}\right]_{\mathrm{k}+1}^{\mathrm{n}}-\left(\frac{\partial \mathrm{H}}{\partial \mathrm{t}}\right)_{\mathrm{k}}^{\mathrm{n}}\right]=-\mathrm{M}^{\mathrm{n}}\left(\frac{\partial \mathrm{H}}{\partial \mathrm{t}}\right)_{\mathrm{k}}^{\mathrm{n}}+\mathrm{F}^{\mathrm{n}}
$$

where $\mathrm{k}=0,1, \ldots$ is the iteration number, $\mu$ is the iteration parameter that will be determined below and $\mathrm{R}=\mathrm{E}-\Delta$ is the positive and self-adjoint operator. We also have the following estimations for R and M (Konshin, 1984):

$$
\alpha(\mathrm{R} \mathrm{f}, \mathrm{f}) \leq(\mathrm{M} \mathrm{f}, \mathrm{f}) \leq \beta(\mathrm{Rf}, \mathrm{f})
$$

where $\alpha=1+\varepsilon \min (\mathrm{H}), \beta=1+\varepsilon \max (\mathrm{H})$, and ( $\mathrm{f}, \mathrm{f})=\int \mathrm{f} \mathrm{R} \mathrm{f} \mathrm{dx} \mathrm{dy.}$

It should be emphasized that $R$ is energetically equivalent to $M$ with constant $\alpha$ and $\beta$ but $R$ is much simpler than $M$. The optimal value of $\mu$ is equal to $\frac{2}{(\alpha+\beta)}$. A direct method is used to inverse $\mathrm{R}$ which includes the Fourier transformation along the $\mathrm{y}$-direction and the standard method for the inversion of a tridiagonal matrix along the $\mathrm{x}$-direction. The choice of $\mathrm{R}$ and $\mu$ provides the rapid convergence of the iterations. The calculations are 5 to 10 times faster than with the widely used over-relaxation method.

3) The value of $\mathrm{H}$ at the $(\mathrm{n}+1)$ level is obtained from 


$$
H^{n+1}=H^{n-1}+2 \Delta T\left(\frac{\partial H}{\partial t}\right)^{n}
$$

where $\Delta \mathrm{T}$ is the time step. The calculation is restarted from $\mathrm{H}^{\mathrm{n}+1}=\frac{1}{2}\left(\mathrm{H}^{\mathrm{n}}+\mathrm{H}^{\mathrm{n}+1}\right)$ after 50 or 100 time steps, as in the paper of Matsuura and Yamagata (1982).

\section{DIPOLE EVOLUTION AND DECAY}

The results of two groups of numerical experiments are presented in this paper. In this section, we consider the first group wherein we investigate the evolution and the decay of isolated dipole eddies with resting background. The initial condition is taken in the form of the Larichev-Reznik soliton:

$$
\begin{gathered}
H=\left[\frac{(1+c) a J_{1}(k r)}{k^{2} J_{1}(k a)}-\frac{r}{k^{2}}\left(1+c+c k^{2}\right)\right] \sin \theta \quad \text { for } r<a \\
H=-\frac{c a K_{1}(p r)}{K_{1}(p a)} \sin \theta \quad \text { for } r \geq a
\end{gathered}
$$

where a is the radius of the soliton, $\mathrm{p}=((1+\mathrm{c}) / \mathrm{c})^{1 / 2}$ is a parameter, and $\theta$ is the polar angle. The values of $\mathrm{k}$ and $\mathrm{p}$ are related by

$$
\frac{\mathrm{J}_{2}(\mathrm{ka})}{\mathrm{ka} J_{1}(\mathrm{ka})}=-\frac{\mathrm{K}_{2}(\mathrm{pa})}{\mathrm{p} \mathrm{a} \mathrm{K}_{1}(\mathrm{pa})}
$$

where $J_{1}, J_{2}$ are the Bessel functions of the first kind and $K_{1}, K_{2}$ are the McDonald functions.

For $\varepsilon=0$, the QGE (1.2) has an exact solution which is simply the initial distribution (4.1) moving at a constant speed c. Some test computations made with $\varepsilon=0$ show that the numerical solution is in good agreement with the theoretical one.

We present here the results of 6 experiments with $\varepsilon=0.01$. The radius of the soliton, a, varies from $3^{1 / 2}$ to 2 , the fluctuation amplitude is $\varepsilon \mathrm{H}_{*}=0.32$ or $\varepsilon \mathrm{H}_{*}=0.65$. The grid includes from $40 \times 50$ up to $120 \times 60$ points. The space steps in the first four cases are $\Delta x=\Delta y=0.3464$. In the last two cases, we use $\Delta x=\Delta y=4$. In the first two cases, the following parameters are used: case $\mathrm{A}, \mathrm{a}=3^{1 / 2}, \mathrm{p}=1.054, \varepsilon \mathrm{H}_{*}=0.32$; case $\mathrm{B}, \mathrm{a}=3^{1 / 2}, \mathrm{p}=0.974, \varepsilon \mathrm{H}_{*}=0.65$.

In cases A and B (Fig. 1), the center of the cyclone is placed at a higher latitude than that of the anticyclone. The vortex pair moves first to the east, in agreement with the quasigeostrophic theory. Later, the dipole deviates towards the equator and it begins to move along a curvilinear trajectory. The cyclone turns around the anticyclone. After some time, the cyclone is intensified, the anticyclone is decreased and the anticyclone turns around the cyclone. The distance between the centers of the vortices is slightly increased and the dipole propagation speed is decreased.

The comparison of cases A and B shows that the larger the amplitude of the vortex, the stronger it deviates from the zonal trajectory. The computations were carried out up to 3.5 


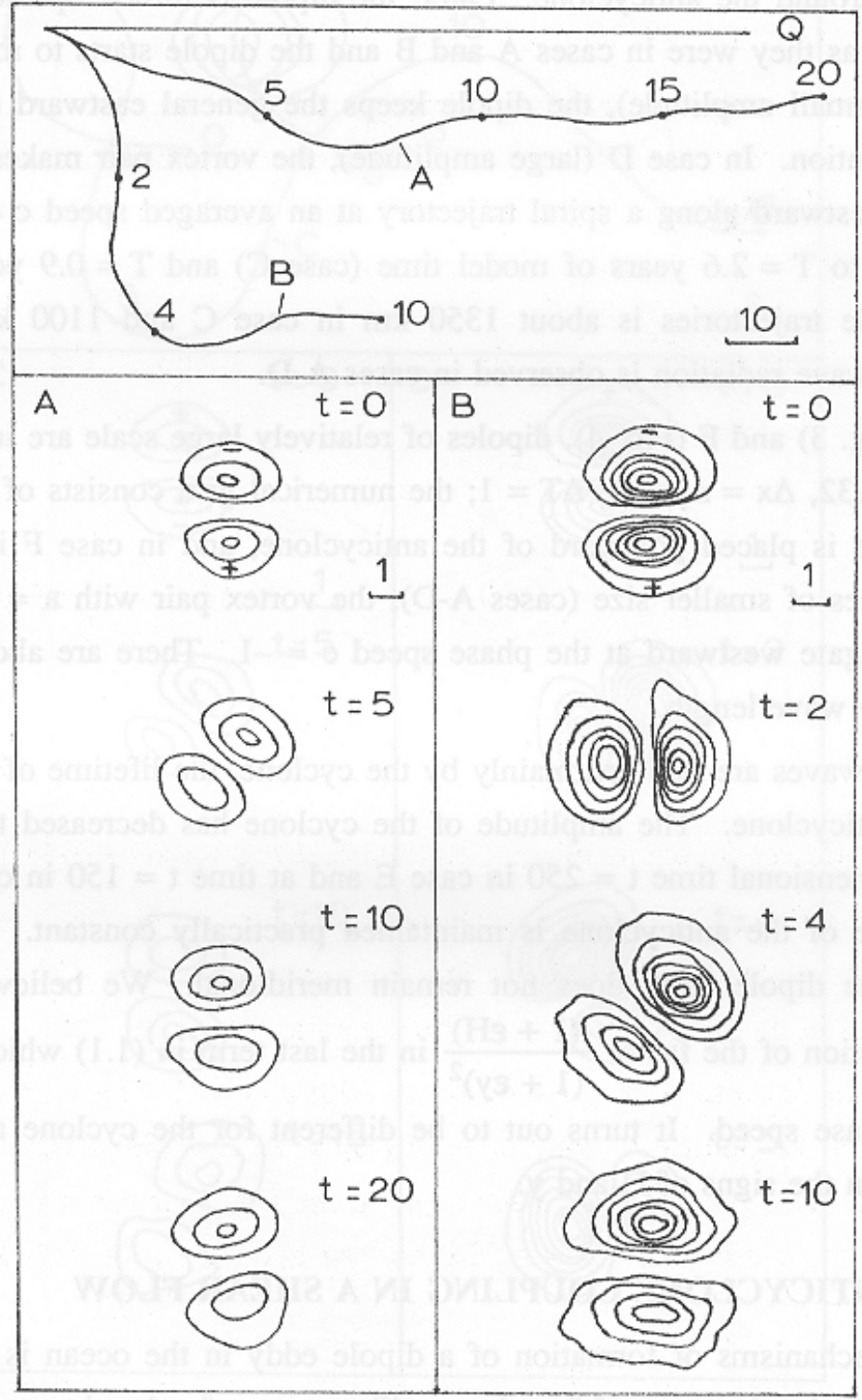

Fig. 1: Trajectories of dipole eddies and time sequence of contour maps of the thickness of the active layer for cases A and B. The positions of the dipole centers at different times are shown. The straight line $(\mathrm{Q})$ represents dipole trajectories according to the quasi-geostrophic model. Maps of $\mathrm{H}(\mathrm{x}, \mathrm{y}, \mathrm{t})$ are shown in the lower panels. Contour interval $\delta \mathrm{H}$ is 10 . Length scales are shown for each case. 
years of model time for case $\mathrm{A}$ and up to 1.7 years for case $\mathrm{B}$. Values of $\beta=1.2 \times 10^{-13} \mathrm{~cm}^{-1} \mathrm{~s}^{-1}$ and $\mathrm{L}_{\mathrm{R}}=15 \mathrm{~km}$ were used to calculate the dimensional data.

In cases $\mathrm{C}$ and $\mathrm{D}$, we use the same parameters as in cases $\mathrm{A}$ and $\mathrm{B}$ except that the initial locations of the cyclone and the anticyclone are permuted. In both cases, the dipole moves along a curvilinear trajectory again, as shown in Fig. 2. First, the dipole moves to the west and the cyclone turns around the anticyclone. Later, the dipole is turned upside down, so that the vortices are placed as they were in cases $\mathrm{A}$ and $\mathrm{B}$ and the dipole starts to move to the east. In case $\mathrm{C}$ (relatively small amplitude), the dipole keeps the general eastward direction during all the time of computation. In case D (large amplitude), the vortex pair makes many turns and it moves generally westward along a spiral trajectory at an averaged speed $c=-1$. The calculations are made up to $T=2.6$ years of model time (case $C$ ) and $T=0.9$ years (case D). The length of the dipole trajectories is about $1350 \mathrm{~km}$ in case $\mathrm{C}$ and $1100 \mathrm{~km}$ in case $\mathrm{D}$. No noticeable Rossby wave radiation is observed in cases A-D.

In cases $\mathrm{E}$ (Fig. 3) and F (Fig. 4), dipoles of relatively large scale are investigated: $a=20$, $\mathrm{p}=0.055, \varepsilon \mathrm{H}_{*}=0.32, \Delta \mathrm{x}=\Delta \mathrm{y}=4, \Delta \mathrm{T}=1$; the numerical grid consists of $120 \times 60$ points. In case $\mathrm{E}$, the cyclone is placed poleward of the anticyclone, and in case $\mathrm{F}$ it is vice versa. In contrast to the eddies of smaller size (cases A-D), the vortex pair with $\mathrm{a}=20$ radiates Rossby waves which propagate westward at the phase speed $c=-1$. There are about four grid points on a typical Rossby wave length.

Since Rossby waves are radiated mainly by the cyclone, the lifetime of the latter is shorter than that of the anticyclone. The amplitude of the cyclone has decreased to half of its initial value at the nondimensional time $\mathrm{t}=250$ in case $\mathrm{E}$ and at time $\mathrm{t}=150$ in case $\mathrm{F}$. During that time, the amplitude of the anticyclone is maintained practically constant. It should be noted that the axis of the dipole eddy does not remain meridional. We believe that this can be explained by the action of the factor $\frac{(1+\varepsilon H)}{(1+\varepsilon y)^{2}}$ in the last term in (1.1) which can be treated as the perturbation phase speed. It turns out to be different for the cyclone and the anticyclone due to differences in the signs of $\mathrm{H}$ and $\mathrm{y}$.

\section{CYCLONE-ANTICYCLONE COUPLING IN A SHEAR FLOW}

One of the mechanisms of formation of a dipole eddy in the ocean is the instability of a jet that results in the development of "mushroom-like currents". Another possibility is the coupling of originally monopole eddies. This situation seems to take place for example in the Kuroshio - Oyashio region of the North Pacific.

In a second group of numerical experiments, we have simulated the interaction and coupling of monopole eddies of equal amplitudes and different signs in a shear flow. Initially, the monopoles were placed sufficiently far from each other. Then, the shear flow moved the eddies to within a short distance from each other. The main goal was to investigate the possibility of coupling of monopole eddies. 


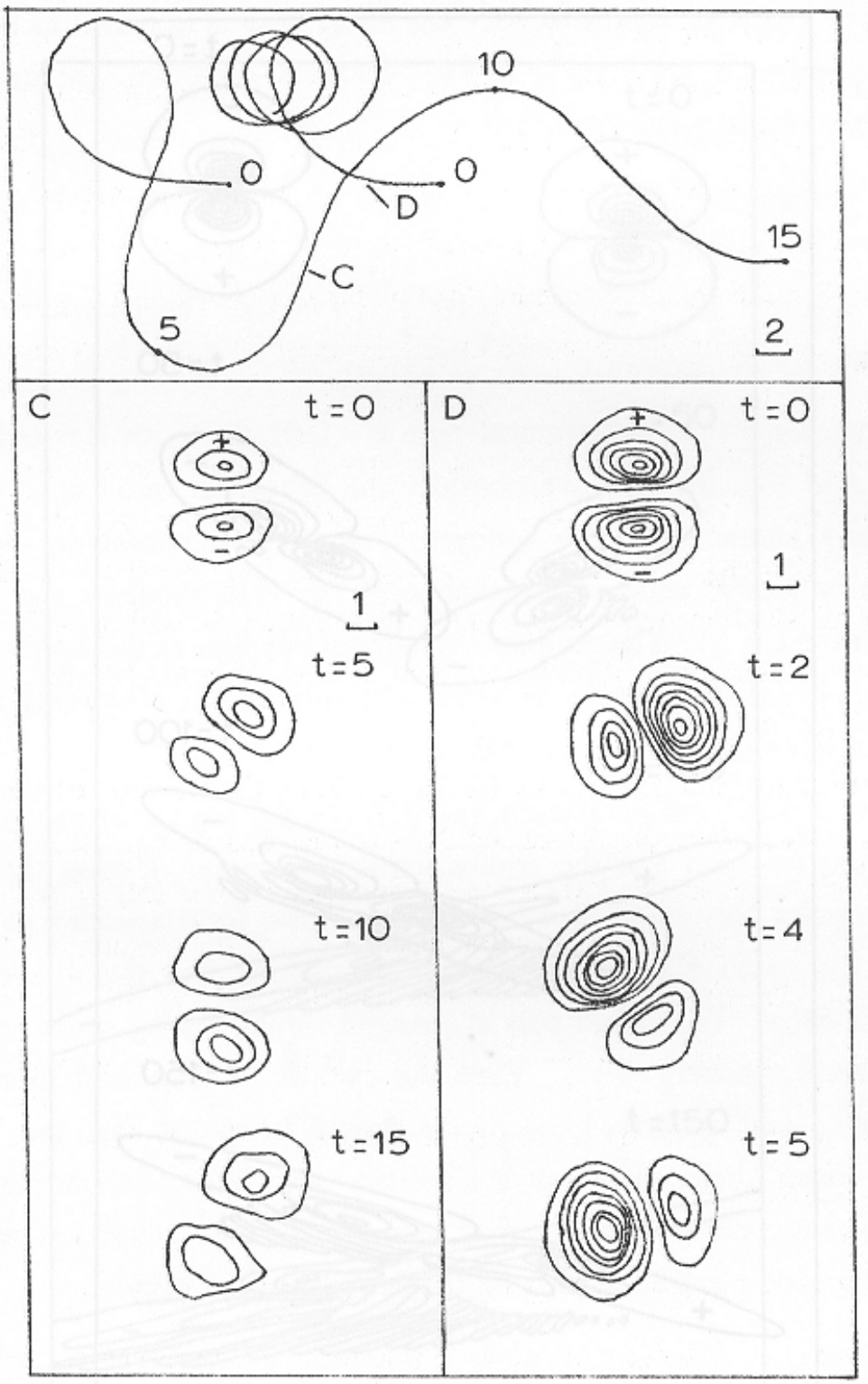

Fig. 2: Same as Fig. 1 for cases C and D. 


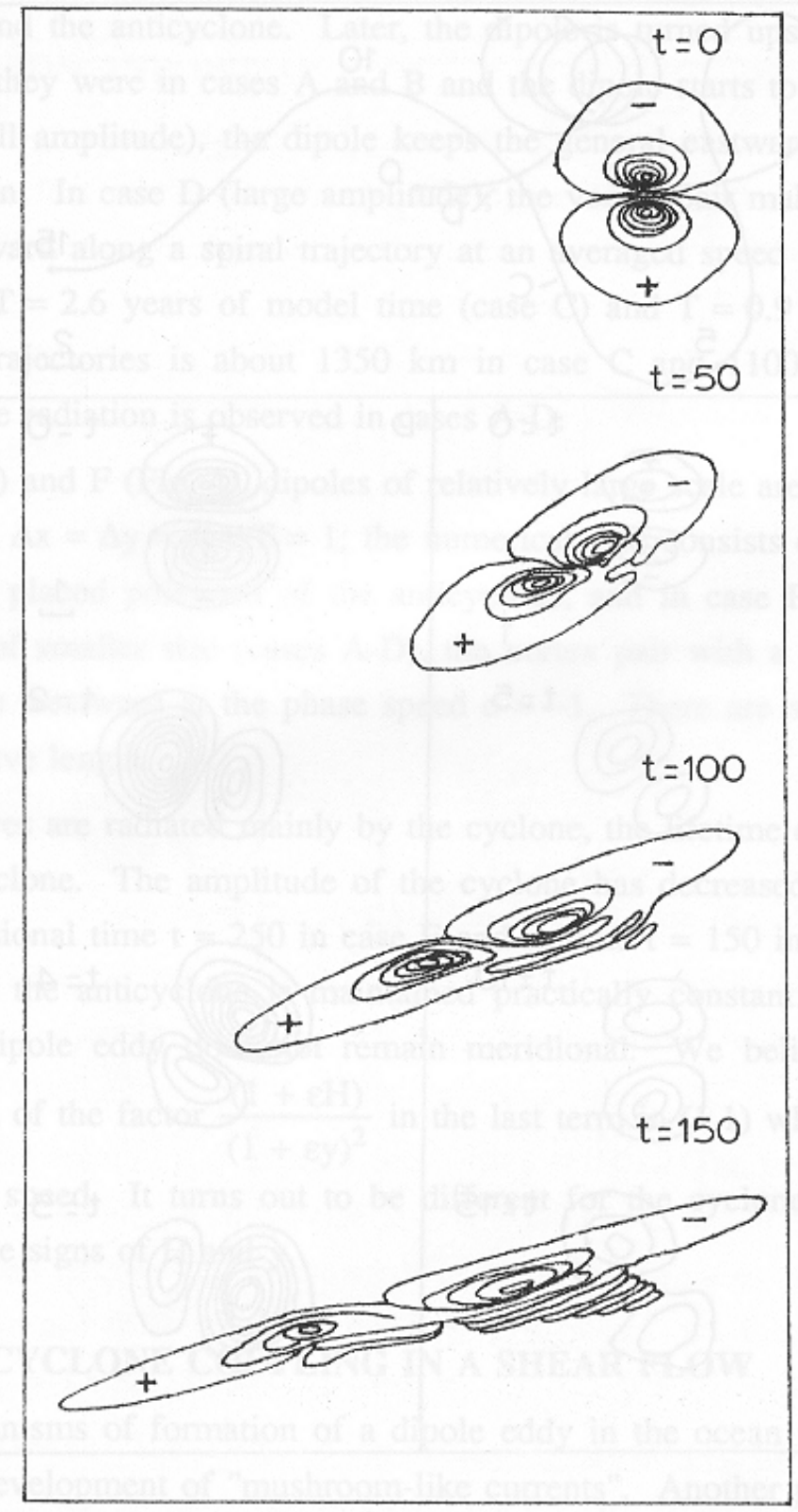

Fig. 3: Time sequence of contour maps for case E. Note that the cyclone radiates Rossby waves more intensively than the anticyclone. 


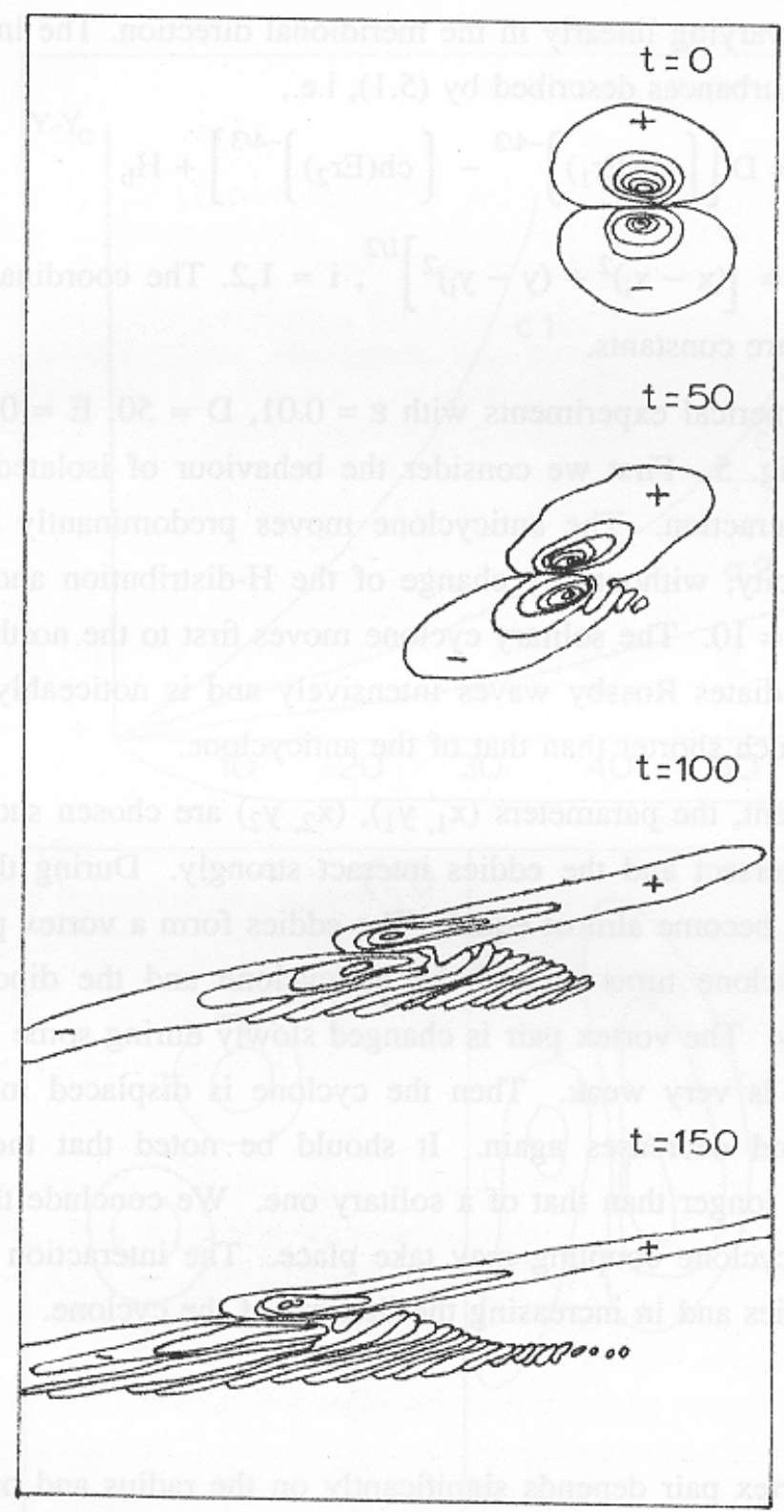

Fig. 4: Same as Fig. 3 for case F. 
As an initial condition for an anticyclone, we use the distribution of $\mathrm{H}$ found in laboratory experiments by Nezlin (1986), which can be well approximated analytically by:

$$
\mathrm{H}=\mathrm{D}(\operatorname{ch}(\mathrm{Er}))^{-4 / 3}
$$

where $\mathrm{D}>0$ and $\mathrm{E}>0$ are the fitting parameters, and $\mathrm{r}$ is the radius. We use the same formula for a cyclone with negative values for D. The shear flow is assumed to be zonally homogeneous, the zonal velocity varying linearly in the meridional direction. The initial field of $\mathrm{H}$ is taken as the sum of two disturbances described by $(5.1)$, i.e.,

$$
\mathrm{H}=\mathrm{D}\left[\left[\operatorname{ch}\left(\mathrm{Er}_{1}\right)\right]^{-4 / 3}-\left[\operatorname{ch}\left(\mathrm{Er}_{2}\right)\right]^{-4 / 3}\right]+\mathrm{H}_{\mathrm{b}}
$$

where $H_{b}=A\left(y_{b}^{2}-y^{2}\right), r_{i}=\left[\left(x-x_{i}\right)^{2}+\left(y-y_{i}\right)^{2}\right]^{1 / 2}, i=1,2$. The coordinates of the monopoles are $\left(\mathrm{x}_{\mathrm{i}}, \mathrm{y}_{\mathrm{i}}\right)$; $\mathrm{A}$ and $\mathrm{y}_{\mathrm{b}}$ are constants.

The results of two numerical experiments with $\varepsilon=0.01, \mathrm{D}=50, \mathrm{E}=0.43, \mathrm{y}_{\mathrm{b}}=20$, and $\mathrm{A}=0.1$ are presented in Fig. 5. First we consider the behaviour of isolated monopoles in a shear flow without any interaction. The anticyclone moves predominantly westward with a very small meridional velocity, without any change of the $\mathrm{H}$-distribution and without Rossby wave radiation up to time $t=10$. The solitary cyclone moves first to the north-west and then it deviates to the north. It radiates Rossby waves intensively and is noticeably decreased. The lifetime of the cyclone is much shorter than that of the anticyclone.

In the second experiment, the parameters $\left(x_{1}, y_{1}\right),\left(x_{2}, y_{2}\right)$ are chosen such that the trajectories of the monopoles intersect and the eddies interact strongly. During the interaction the velocities of the monopoles become almost equal. The eddies form a vortex pair which moves to the west. Then, the cyclone turns around the anticyclone and the dipole axis becomes directed almost meridionally. The vortex pair is changed slowly during some time period. The radiation of Rossby waves is very weak. Then the cyclone is displaced more poleward. It begins to radiate waves and decreases again. It should be noted that the lifetime of the interacting cyclone is much longer than that of a solitary one. We conclude that under definite circumstances, cyclone-anticyclone coupling may take place. The interaction results in changing the trajectory of the eddies and in increasing the lifetime of the cyclone.

\section{DISCUSSION}

The evolution of a vortex pair depends significantly on the radius and on the intensity of the vortices. Modons of small amplitude propagate predominantely in a zonal direction in agreement with the quasi-geostrophic theory. If the intensity of the vortices is high and the radius comparable to the radius of deformation, the modon basically retains its inner structure but the trajectory becomes rather entangled. Dipole eddies of large size and amplitude do not keep their initial structure and radiate waves, their trajectories being almost zonal. 


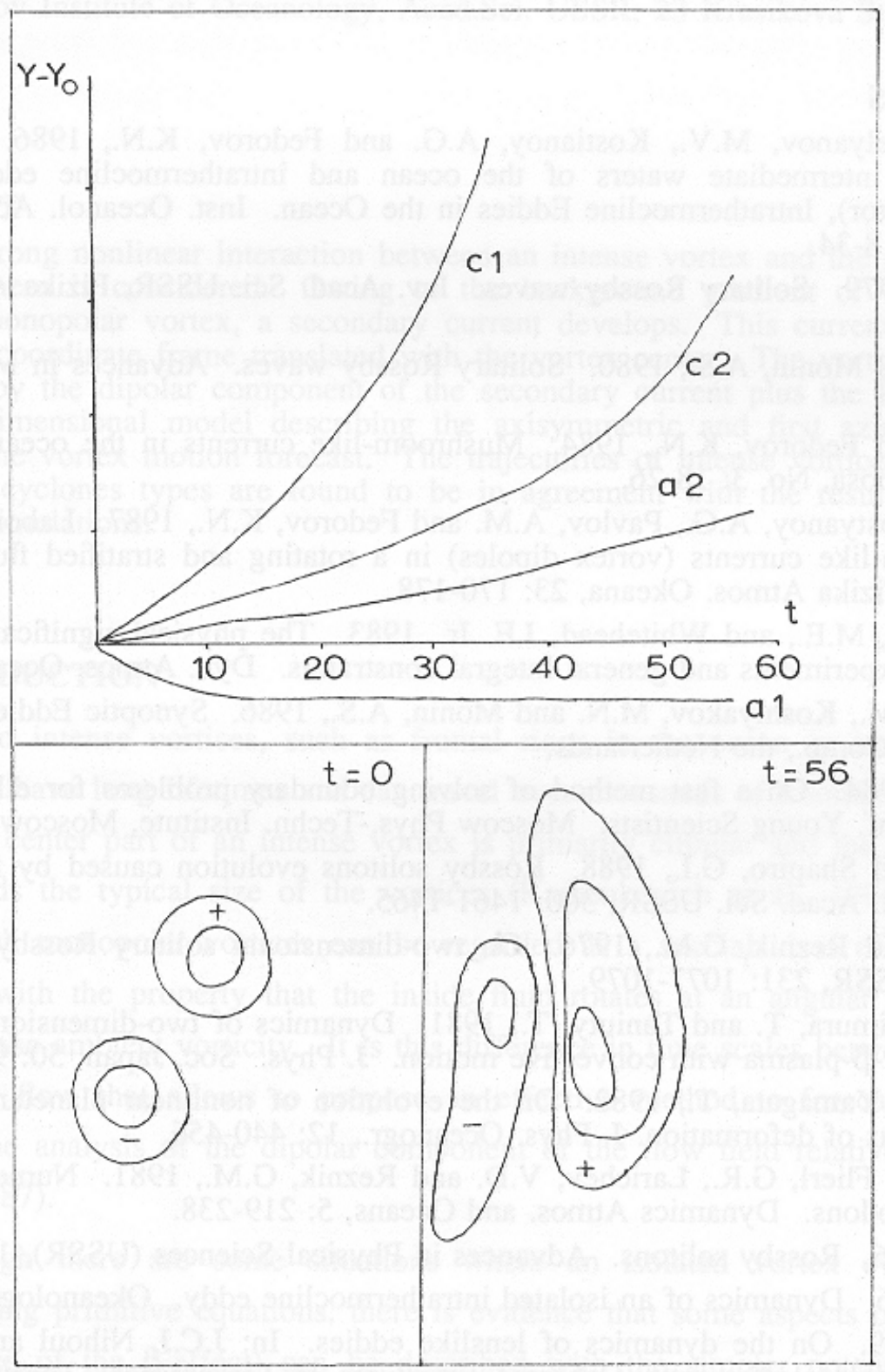

Fig. 5: Cyclone-anticyclone interaction and coupling in a shear flow. The meridional displacement of isolated (c1, a1) and interacting (c2, a2) cyclones and anticyclones is shown at the top. The lower panels show the contour maps of the thickness disturbance, $\mathrm{H}-\mathrm{H}_{\mathrm{b}}$, at $\mathrm{t}=0$ and $\mathrm{t}=$ 56. 
In a shear flow a solitary cyclone has a much shorter lifetime than in a resting fluid. The trajectory of a cyclone has a noticeable meridional component. If the flow brings a cyclone and an anticyclone together, so that they can interact, their velocities are equalized and a coupling may occur. Our numerical experiments show that intensive vortex pairs are stable with respect to the action of additional nonlinear forces which are not taken into account in the standard quasi-geostrophic model. This conclusion is in correlation with the wide occurance of

\section{REFERENCES}

Belkin, I.M., Emelyanov, M.V., Kostianoy, A.G. and Fedorov, K.N., 1986. Thermohaline
structure of Fedorov (Editor), Intrathermocline of the ocean and intrathermocline eddies. In: K.N. Moscow, pp. 8-34.

Berestov, A.L., 1979. Solitary Rossby waves. Izv. Acad. Sci. USSR, Fizika Atmos. Okeana,
15: 648-654.

Berestov, A.L. and Monin, A.S., 1980. Solitary Rossby waves. Advances in Mechanics, 3: 3-
34. Ginsburg, A.I. and Fedorov, K.N., 1984. Mushroom-like currents in the ocean. Issledovanie
semli iz kosmosa, No. 3: 18-26.

Ginsburg, A.I., Kostyanoy, A.G., Pavlov, A.M. and Fedorov, K.N., 1987. Laboratory modeling of mushroom-like currents (vortex dipoles) in a rotating and stratified fluid. Izv. Acad.

Flierl, G.R Stern, ME and Whiteled, $170-178$.

Laboratory experiments and genead, J.E. Jr., 1983. The physical significance of modons:

Kamenkovich, V.M., Koshlyakov, M.N. and constraints. Dyn. Atmos. Oceans. 7: 233-263. Reidel Publ. Comp., the Netherlands.

Konshin, V.N., 1984. On a fast method

Proc. 9th Conf. Young Scientists.

Konshin, V.N. and Shapiro, G.I., 1988. Row Phys.-Techn. Institute, Moscow, pp. 128-133. effects. Dokl. Acad. Sci. USSR, 300: 1461-1465.

Larichev, V.D. and Reznik, G.M., 1976. On two-1465. Acad. Sci. USSR, 231: 1077-1079.

Makino, M., Kamimura, T. and Taniuty, T., 1981. Dynamics of two-dimensional solitary vortices in a low- $\beta$-plasma with convective motion. J. Phys. Soc. Japan, 50: 980-989.

Matsuura, T. and Yamagata, T., 1982. On the evolution than the radius of deformation. J. Phys. Oceanogr., 12: 440-456.

McWilliams, J.C., Flierl, G.R., Larichev, V.D. and

barotropic modons. Dynamics Atmos. and Oceans, 5: 219-238. 1981. Numerical studies of

Nezlin, M.V., 1986. Rossby solitons. Adva

(USSR), 150: 3-60.

Shapiro, G.I., 1989. On the dynamics of lentikathermocline eddy. Okeanologiya, 27: 21-27.

(Editors), Mesoscale/Synoptic Coherent Structures in In: J.C.J. Nihoul and B.M. Jamart

Oceanography Series, Elsevier, Amsterdam (this volume).

Williams, G.P., 1985.

1237-1243. 